
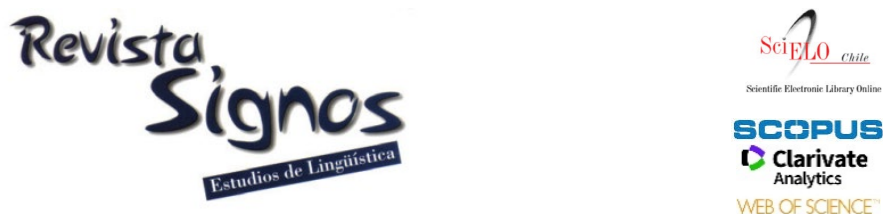

WEB OF SCIENCE

\title{
Análisis de la polisemia de nombres eventivos no deverbales a partir de las propuestas de la Léxico-Gramática y la Teoría del Lexicón Generativo*
}

\author{
Analysis of polysemy of non deverbal names events from the \\ Lexicon-Grammar and the Generative Lexicon Theory
}

\author{
Walter Koza \\ PONTIFICIA UniversidAd CATÓLICA DE VALPARAíso \\ CHILE \\ walter.koza@pucv.cl
}

Recibido: 10-X-2018 / Aceptado: 26-XII-2018

DOI: $10.4067 / \mathrm{S} 0718-09342019000200502$

\section{Resumen}

El problema de la eventividad en las unidades léxicas nominales supo ser abordado con gran interés por la gramática generativa en los años setenta, no obstante, el foco de aquellas investigaciones iniciales estuvo centrado en los sustantivos derivados de verbos. Por el contrario, los estudios sobre los nombres eventivos no deverbales (NEND), o nombres de eventos simples han sido en gran parte escasos. A tales efectos, el objetivo del presente trabajo es establecer un análisis de la polisemia de un grupo de NEND pertenecientes al ámbito de la medicina, a partir de la propuesta de la LéxicoGramática (LG) (Gross, 1975) y una posterior articulación con la Teoría del Lexicón Generativo (TLG) (Pustejovsky, 1995). Para ello, se contemplan los siguientes pasos metodológicos: (i) elaboración de tablas LG de preferencias de selección léxica; (ii) elaboración de tablas LG de posibilidades transformacionales de las estructuras argumentales (EA) resultantes del análisis distribucional de (i); (iii) análisis de las EA que seleccionan los NEND en calidad de argumentos, y (iv) organización de Estructuras Semánticas (ES) para las unidades léxicas analizadas, según los criterios de la TLG. Los resultados permiten observar, por un lado, que la propuesta de la LG permite dar cuenta de los sentidos eventivos de los NEND, no obstante, es necesario extender la metodología propuesta por la LG a la EA para otras posibilidades de significado. Asimismo, por otro lado, se evidencia que la eventividad es un fenómeno sintáctico que surge mediante un mecanismo de co-composición de las unidades léxicas infraespecificadas.

Palabras Clave: Nombres eventivos no deverbales, léxico-gramática, Teoría del Lexicón Generativo, polisemia, estructura argumental. 


\begin{abstract}
The issue with eventivity in nominal lexical units was approached with great interest by the generative grammar in the seventies; however, the focus of those first investigations was centered on nouns derived from verbs. On the contrary, studies about nondeverbal eventive nouns (NDEN) or simple event nouns have been largely scarce. For this purpose, the objective of this work is to stablish an analysis of the polysemy of a NDEN group belonging to the medical field, based on the Lexicon-Grammar (LG) proposal (Gross, 1975) and a subsequent articulation with the Generative Lexicon Theory (TLG) (Pustejovsky, 1995). For this, the following methodological steps are considered: (i) development of LG tables of lexical selection preferences; (ii) elaboration of LG tables of transformational possibilities of the argument structures (AE) resulting from the distributional analysis of (i); (iii) analysis of the AEs that select NDENs as arguments, and (iv) organization of Semantic Structures (SE) for the lexical units analyzed, according to the TLG criteria. The results allow observing, on one hand, that the LG proposal allows to account for the eventual meanings of NDEN; nevertheless, it is necessary to expand the methodology proposed by the LG to the AE in order to cover other possibilities of meaning. Likewise, on the other hand, it is demonstrated that eventivity is a syntactic fenomenon that arises through a mechanism of co-composition of the infraspecified lexical units.
\end{abstract}

Key Words: Deverbal eventive nouns, lexicon-grammar, Generative Lexicon Theory, polysemy, argument structure.

\title{
INTRODUCCIÓN
}

El problema de la eventividad en las unidades léxicas nominales ha sido abordado con gran interés por la gramática generativa en los años setenta, no obstante, el foco de aquellas investigaciones iniciales estuvo centrado en los sustantivos derivados de verbos del tipo de 1.a. (Resnik, 2010). Dichos trabajos consideraban, por un lado, las propiedades intrínsecas del léxico que se proyectan en la sintaxis (Chomsky, 1970) y, por otro, las limitaciones de las propias categorías gramaticales respecto del fenómeno de la eventividad. No obstante, los estudios sobre los nombres eventivos no deverbales, o nombres de eventos simples (Grimshaw, 1990), como los de 1.b., han sido en gran parte escasos y, generalmente, considerados dentro de los tipos de nombres complejos (Pustejovsky, 1995). Estas unidades léxicas no son derivadas de verbos, no obstante, tienen la particularidad de requerir argumentos y, por ende, proyectar estructura argumental (EA).

(1) a. destrucción: la destrucción de la ciudad por los extraterrestres merecimiento: el merecimiento de una vida mejor por parte de los trabajadores recorrida: la recorrida por los barrios marginales

b. boda: Juan y María tuvieron su boda. sorpresa: El hijo le dio una sorpresa a la madre. fiaca: Juan hace fiaca (en horas de trabajo). 
Los nombres eventivos no deverbales (NEND) -al igual que las nominalizaciones eventivas- recurren a verbos soportes que los actualizan y, de esa manera, pueden desarrollar, además de una EA, una estructura eventiva (EE) (Gross, 2014). A tales efectos, la diferencia principal, de acuerdo con Resnik (2010), no estaría dada en la EE, sino en virtud de su estructura funcional y en relación con condiciones de realización de argumentos en el sintagma determinante (SD) ('la destrucción de la ciudad por parte de Juan’ vs. *'la cesárea de la paciente por parte del médico').

Respecto de los verbos soportes, estos son definidos en el marco de la LG como aquellos que, o bien, carecen de posibilidad de predicar (como el caso de 'ser') o la pierden total o parcialmente. A su vez, la función de los verbos soporte es actualizar un predicado nominal o adjetival (Gross, 2014), que consiste en aportar las marcas de flexión, de las que carece el predicado, y asignarle caso nominativo al sujeto. No obstante, es pertinente la observación de De Miguel (2011), quien señala que estos verbos, tanto en su uso pleno como en calidad de soportes, poseen una definición mínima en la que los rasgos subléxicos concuerdan con los NEND a los que actualizan.

Ahora bien, a fin de vislumbrar las posibilidades de significado de los NEND, resulta de interés la metodología propuesta por la Léxico-Gramática (LG), de Gross $(1975,1998)$, que establece que las lenguas naturales deben describirse a partir del conjunto de oraciones simples que las componen. Para ello, propone la confección de tablas que reúnen elementos léxicos de una categoría gramatical que comparten propiedades (Tolone, 2012). En sus primeras propuestas (Gross, 1975), la LG se organiza en tres tipos:

- Una LG para verbos predicativos.

- Una LG para verbos soporte.

- Una LG para estructuras fijas del tipo 'meter la pata', 'salvar el pellejo'.

Se han elaborado propuestas de análisis basadas en la LG, en su gran mayoría, para el francés, fundamentalmente en relación con el análisis automático (Gross, 1998; Gross, 2014; Silberztein, 2016). Asimismo, también pueden mencionarse propuestas para el italiano (Langella, 2016), el griego (Papadopoulou, 2010), el croata (Kocijan \& Librenjak, 2016) y el portugués (Mota, Barreriro, Raposo, Ribeiro, Curto \& Coheur, 2016), entre otras. No obstante, se evidencia una escasez de trabajos para el español, con excepción de los llevados a cabo por Lamiroy (1991), Subirats (1987), Blanco (2016) y Koza, Filippo, Cotik, Stricker, Muñoz, Rivas, Godoy y Martínez (2018).

Por otro lado, se hace pertinente estructurar la información obtenida en el análisis proporcionado por la LG a fin de que el fenómeno no se reduzca a una enumeración de sentidos, sino que, por el contrario, proporcione evidencia empírica a un modelo organizativo de las entradas léxicas. A tales efectos, el presente trabajo articula la LG 
con la Teoría del Lexicón Generativo (TLG) (Pustejovsky, 1995, 2011, 2013; De Miguel, 2009; Berri, 2012; Adelstein \& Boschiroli, 2015). La TLG da cuenta de las entradas léxicas en relación con los sentidos que estas generan. Para ello, postula una única estructura semántica infraespecificada para cada unidad, junto con una serie de mecanismos generativos.

A tales efectos, el objetivo del presente trabajo es establecer un análisis de la polisemia de los NEND a partir de la LG (Gross, 1975, 1998) y una posterior articulación con la TLG (Pustejovsky, 1995, 2011, 2013); esto último, focalizado en el análisis de los nombres complejos (Rumshisky, Grinberg \& Pustejovsky, 2007; Romero, 2015). Para ello, se contemplan los siguientes pasos metodológicos: (i) elaboración de tablas LG de preferencias de selección léxica para los NEND; (ii) elaboración de tablas LG de posibilidades transformacionales de cada EA resultante del análisis distribucional de (i); (iii) análisis de las EA que seleccionan los NEND en calidad de argumentos, y (iv) elaboración de estructuras semánticas (ES), a partir de la información proporcionada en (i), (ii) y (iii), según los criterios de la TLG. En relación con el último ítem, se busca especificar la relación entre (a) los argumentos verdaderos y las clases de objeto; (b) los Qualia que se activan a partir de la presencia de ciertos argumentos, y (c) la proyecciones y restricciones de significado de las propiedades transformacionales.

Los resultados señalan que la articulación de ambas propuestas permite un análisis detallado del fenómeno de la polisemia de este tipo de unidades léxicas. Asimismo, se pudo observar también que algunos de los NEND analizados pueden tener tanto lecturas eventivas como no eventivas, lo que favorecería una perspectiva dinámica de las relaciones léxicas, a partir de la cual se establece, como se sugiere en la TLG, que las palabras carecen de significados cerrados y estables, y que, por el contrario, se trata de unidades infraespecificadas, capaces de ampliarse cuando aparecen en un determinado contexto (De Miguel, 2011).

El artículo se organiza de la siguiente manera. En la sección 1, se presenta el marco teórico que sustenta la propuesta. En 2, se describe la metodología llevada a cabo. En 3 , se presenta el análisis y los resultados obtenidos. Finalmente, en 4 , se presentan las conclusiones derivadas de la investigación.

\section{Marco teórico}

A continuación se presenta el marco teórico, que se organiza de la siguiente manera. En primer lugar, se presentan las propiedades de los NEND del español (Fábregas, 2010; Resnik, 2010). En segundo lugar, se describe el marco de la LG (Gross, 1975, 1998). En tercer lugar, se presenta la propuesta de la TLG (Pustejovsky, 1995, 2011, 2013), y cómo esta se ha empleado en el estudio de nombres complejos (Rumshisky et al., 2007; Romero, 2015). 


\subsection{Propiedades de los nombres eventivos no deverbales}

Dentro de los estudios sobre las unidades léxicas nominales, ha cobrado interés aquellas que remiten a eventos, tanto derivadas de un verbo ('recorrida', 'inauguración', 'compra') como 'puros' (Fábregas, 2010). Se trata de nombres que, a diferencia de los no eventivos, no designan entidades físicas, sino acontecimientos o sucesos (Bosque, 1999). Por otro lado, tienen un comportamiento similar al de los verbos, en la medida en que poseen una capacidad predicativa y proyectan una estructura argumental (Grimshaw, 1990), lo que implica, por ende, la selección de argumentos. No obstante, la diferencia principal radica en que, al carecer de marcas de flexión temporal y de la posibilidad de asignar caso, deben recurrir a un verbo soporte que los actualice (Gross, 2014), como, por ejemplo, 'tener' en 'tener un parto'.

Bosque (1999) señala que este tipo de nombres puede ser sujeto de predicados como 'tener lugar' ('tuvo lugar el parto') u objetos directos de verbos como 'presenciar' ('presenciar el parto'). A la vez, el carácter temporal de estas unidades se evidencia en la posibilidad de ser seleccionados por la preposición 'durante' ('el sexo durante el embarazo') y en construcciones adverbiales con 'antes de' ('antes del parto') y 'después de' ('después del parto'). En este contexto, Nazar, Soto y Urrejola (2017) señalan que se pueden producir ambigüedades a partir de una posible lectura eventiva en nombres que, en principio, no lo serían ('después del último bus'). Dicho fenómeno no es infrecuente, y estarían contemplados en los casos de polisemia sistemática o regular (Pustejovsky, 1995; Adelstein, Berri \& Boschiroli, 2012). En este sentido, en el presente trabajo se asume que la eventividad es un fenómeno que se proyecta en la sintaxis y, en este caso, producto de mecanismos generativos.

En relación con los estudios para el español, dentro del marco generativista, pueden mencionarse el llevado a cabo por Resnik (2010) y la propuesta de Fábregas (2010). Resnik (2010) parte de la propuesta de Alexiadou (2001), enmarcada en la Morfología Distribuida (Halle \& Marantz, 1993) y propone extender el estudio de los nombres de evento a lo que Grimshaw (1990) había clasificado como nombres eventivos simples. En dicho estudio, la autora analiza los contextos sintácticos que permiten diferenciar los nombres eventivos deverbales, los no deverbales, los resultativos y los semelfactivos denominales; lo que le permite establecer diferencias estructurales, que se reflejan en la estructura funcional.

Fábregas (2010), a su vez, propone una división entre los NEND puros y los trasladados. Mientras que los primeros no son producto de procesos morfológicos de derivación, los segundos provienen de otras bases léxicas. El autor señala que deben ser distinguidos de los nombres no eventivos que expresan temporalidad y de las nominalizaciones deverbales. Asimismo, concluye que todos los nombres de evento, puros o trasladados, deben ser contables. 
En el presente trabajo, se siguen los lineamientos de estos dos autores para la selección de NEND analizados.

\subsection{Un estudio integrado de la polisemia: La Léxico-Gramática}

El marco de la LG fue propuesto por Gross $(1975,1998)$ y se basa en tres aspectos principales: (i) el hecho de considerar a la sintaxis indisoluble del lexicón; (ii) la identificación de oraciones simples (en lugar de palabras o sintagmas) como contextos sintácticos y semánticos mínimos de análisis, y (iii) el hecho de ofrecer una formalización y un método descriptivo aplicable a cualquier lengua (Elia, Monteleone \& Marano, 2011). Esta teoría plantea un estudio combinado de las reglas sintácticas y preferencias de selección léxica y en relación con las posibilidades de transformación que tiene una oración determinada.

Para poder llegar a tal descripción, Gross (1975) propone la elaboración de tablas organizadas en clases, que reúnen elementos léxicos de una categoría gramatical que comparten propiedades (Tolone, 2012). Una tabla se presenta sobre la forma de una matriz: (i) en línea, los elementos de la clase correspondiente; (ii) en columna, las propiedades sintáctico-semánticas que no son necesariamente aceptadas por todos los miembros de la clase, y (iii) al cruce de una línea y de una columna el signo + o - en función de si la entrada léxica descrita por la línea acepta o no la propiedad descrita por la columna. Una propiedad sintáctico-semántica es una información que se refiere directamente a la construcción de base asociada a la clase, a una transformación de la construcción de base, o a una construcción adicional. Si una palabra tiene dos sentidos distintos, entonces tendrá dos entradas léxicas. A modo de ejemplo, se presenta en la Figura 1 un fragmento de una de las tablas de verbos adaptadas por Pivaut (1989), presentada en Tolone (2012).

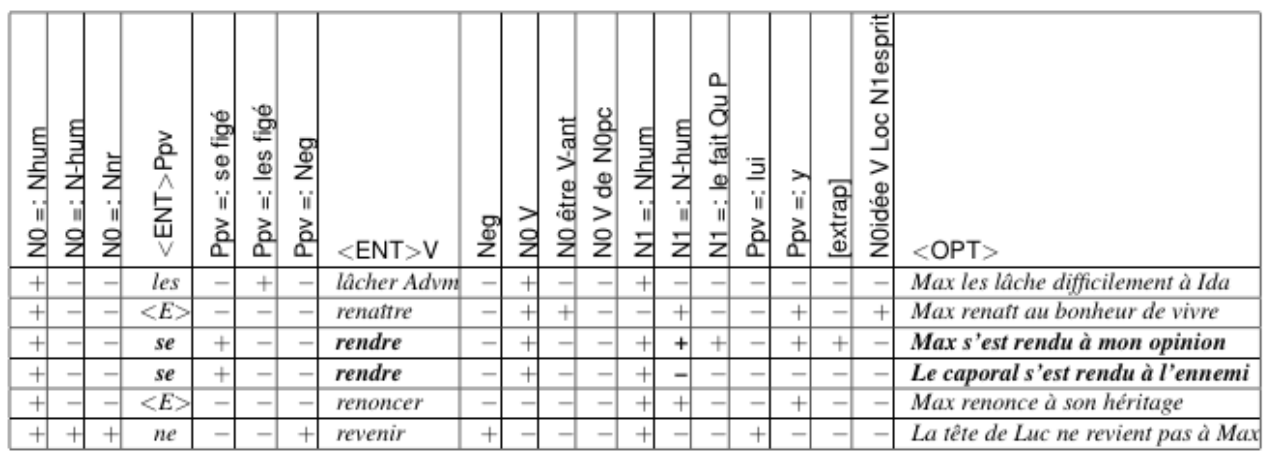

Figura 1. Ejemplo de una tabla según los parámetros de la LG (Tolone, 2012).

Como se puede observar un verbo como rendre ('rendir') va a tener doble entrada según los tipos de objetos que exija ( $\mathrm{N} 0=$ Humano, N1=No Humano: 'Max se rindió a mi opinión'; N0=Humano, N1=Humano: 'El cabo se rindió al enemigo'). 
Desde hace tiempo, se han venido desarrollando diversos estudios a fin de mejorar las tablas propuestas por Gross (Pivaut, 1989; Tolone, 2009, 2012), a la vez que se han propuesto diversas posibilidades de descripción de los predicados (Gross, 2014) y los objetos seleccionados por ellos (Le Pesant \& Mathieu-Colas, 1998).

Este método permite dar cuenta de la polisemia de las entradas léxicas a partir de las distintas EA que proyectan estas. A continuación se ejemplifica en la Tabla 1 con el verbo 'comer':

Tabla 1. Fragmento de una tabla LG de preferencias de selección para el verbo 'comer'.

\begin{tabular}{|c|c|c|c|c|c|}
\hline \multicolumn{2}{|c|}{ Argumento 0 } & \multirow{2}{*}{ Lema } & \multicolumn{2}{|c|}{ Argumento 1 } & \multirow{2}{*}{ Ejemplo } \\
\cline { 4 - 5 } +ANIM & -ANIM & & +ALIMENTO & -ALIMENTO & \multirow{2}{*}{ El perro comió un filete. } \\
\hline+ & - & comer & + & - & El óxido comió el metal. \\
\hline- & + & comer & - & + & \\
\hline
\end{tabular}

Al respecto, pueden observarse dos posibles significados para el lema 'comer': uno referido a la acción de alimentarse y otro relacionado con el proceso de corrosión de un metal. Si bien la naturaleza de los argumentos proporciona pistas para desambiguar los significados en los dos casos, el marco de la LG propone una segunda tabla en la que se listan las transformaciones ${ }^{1}$ que puede tener una estructura argumental y que resulta pertinente para las tareas de desambiguación. Al respecto, en Messina y Langella (2015) se mencionan dentro de las transformaciones posibles a la pronominalización (2), la reducción de oración (3), la nominalización (4 y 5), la adjetivación (6 y 7) y la paráfrasis pasiva (8).

(2) 01: La médica diagnosticó el cáncer $\rightarrow$ 02: Ella lo diagnosticó.

(3) 01: El hecho de que el cáncer avance preocupa a la médica. $\rightarrow$ 02: El cáncer preocupa a la médica.

(4) 01: El hecho de que el cáncer avance preocupa a la médica. $\rightarrow$ 02: El hecho de que el cáncer avance es una preocupación para la médica.

(5) 01: El hecho de que el cáncer avance preocupa a la médica. $\rightarrow$ 02: El hecho de que el cáncer avance genera preocupación en la médica.

(6) 01: El hecho de que el cáncer avance preocupa a la médica. $\rightarrow$ 02: El hecho de que el cáncer avance es preocupante para la médica.

(7) 01: El hecho de que el cáncer avance preocupa a la médica. $\rightarrow$ 02: El hecho de que el cáncer avance hace que la médica esté preocupada.

(8) 01: Él cáncer afecta al paciente. $\rightarrow$ 02: El paciente es afectado por el cáncer. 
La postulación de transformaciones que permiten pasar de una oración 01 a una oración 02 son justificadas a través de un argumento de economía: 01 posee determinadas restricciones, bastante complejas, que aparecen también en 02 , lo que implica que, si se las describe de manera independiente, esas restricciones aparecerían dos veces en la gramática. A tales efectos, la solución transformacional consiste en la descripción de 01 con todas sus restricciones y en la aplicación de una operación que transforma a 01 en 02, sin afectar a estas (Palma, 1994). No obstante, de acuerdo con Gross (1975), todas las transformaciones tienen excepciones, por lo que es indispensable diferenciar los elementos del léxico, según puedan o no sufrir una determinada transformación y para ello, es requisito indispensable contar con una descripción de estas unidades y de sus propiedades, a fin de determinar clases sintácticas.

De este modo, continuando con el ejemplo de 'comer', se puede elaborar una tabla de transformaciones como la siguiente:

Tabla 2. Ejemplo de Tabla LG de transformaciones.

\begin{tabular}{|c|l|l|}
\hline \multicolumn{1}{|c|}{ Oración } & Voz Pasiva & Nominalización \\
\hline El perro comió un filete. & + & + \\
\hline El óxido comió el metal. & + & - \\
\hline
\end{tabular}

De este modo, se puede diferenciar el significado de ambas oraciones a partir de las posibilidades de transformación que presentan:

(9) a. 01: El perro comió un filete $\rightarrow$ 02: La comida del perro fue un filete.

b. 01: El óxido comió el metal $\rightarrow 02: *$ La comida del óxido fue el metal.

Por otro lado, en relación con los significados que se generan a partir de la EA, y las posibilidades de desambiguación, cuando una serie de argumentos, en una misma posición dada, le otorgan a un predicado un significado constante, estos constituyen lo que Gross (2014) denomina 'clases de objeto'. Por ejemplo, nombres como 'vino', 'cerveza', 'agua' o 'Coca-Cola' forman una clase objeto para el verbo 'tomar' en la medida en que le dan a este el mismo significado (equivalente a 'beber'); dicha clase podría denominarse 'bebidas'. Por otro lado, nombres como 'tren', 'bus', 'avión' constituirían otra clase de objetos ('medios de transporte'), que le otorgarían otro significado al verbo (ver Figura 2). 


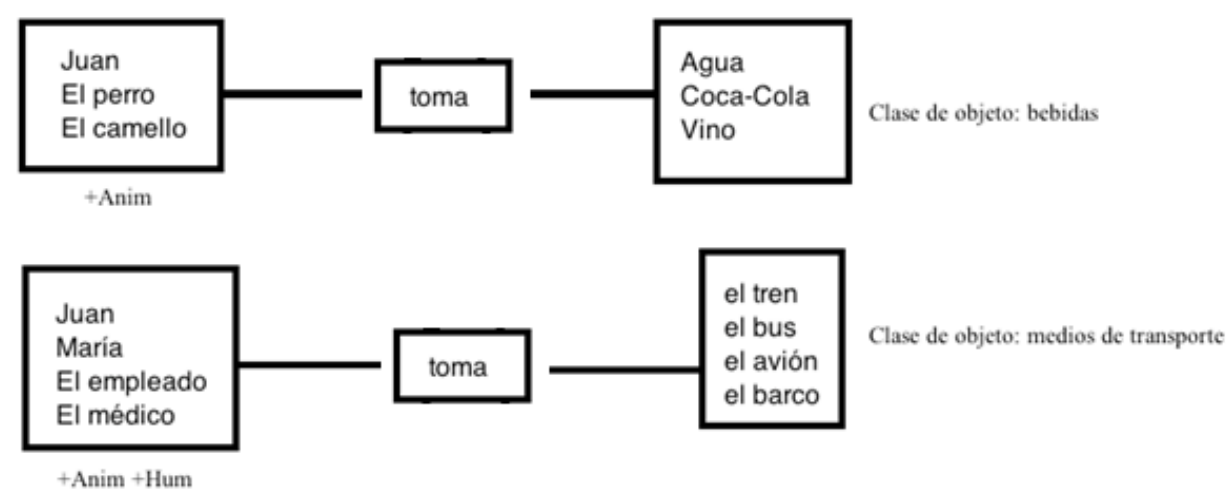

Figura 2. Ejemplos de Clases de objeto.

Un mismo sustantivo puede pertenecer a más de una clase de objeto, según sea el predicado que lo seleccione. De este modo, 'Coca-Cola', por ejemplo, va a pertenecer a la clase de objetos de 'líquidos + bebibles' y a la de 'mercaderías', puesto que puede ser seleccionado por verbos como 'comprar'o 'vender'.

Las preferencias de selección léxica permiten compilar clases de objeto. En el presente trabajo se analizan predicados propios del área de la medicina, específicamente, ginecología y obstetricia, y se establecieron como clases de objeto: 'profesionales de la medicina' (ProfMed: +Animado+Humano+Profesional Médico); 'pacientes' (+Animado+Humano); 'zona anatómica', entre otros.

\subsection{El análisis de los nombres complejos en la Teoría del Lexicón Generativo}

A fin de organizar los datos arrojados por el análisis de la LG, como se mencionó más arriba, el presente trabajo toma en consideración la propuesta de la TLG (Pustejovsky, 1995; De Miguel, 2009), con especial atención a las propuestas de análisis de los denominados nombres de tipo complejo (Pustejovsky, 1995; Rumshisky et al., 2007; Romero, 2015).

La TLG establece un modelo organizacional de las entradas léxicas en relación con los significados que estas puedan generar a partir de la combinación con otras unidades. Un ejemplo típico sería el adjetivo 'rápido' en 'un jugador rápido’ y 'un juego rápido'. Para ello, este marco plantea que existe un significado mínimo y una posibilidad regida por principios y mecanismos regulares que desencadenan múltiples significados en contexto. Para ello, la teoría plantea la existencia de cuatro niveles de representación que estructuran dicha información:

(i) La estructura argumental, que especifica el número de argumentos de un predicado, la clase semántica y el modo en que se realizan sintácticamente.

(ii) La estructura eventiva, que se indica el tipo de evento denotado por un predicado. 
(iii) La estructura de Qualia, que incluye información sobre las características fundamentales de la entidad a la que se refiere, relacionando las estructuras eventiva y argumental. Se incluyen en ella:

- el Quale constitutivo, que codifica la relación entre un objeto y sus partes;

- $\quad$ el Quale formal, que codifica aquello que distingue el objeto dentro de un dominio más extenso;

- el Quale télico, que codifica el propósito y la función del objeto, y

- el Quale agentivo, que codifica los factores implicados en el origen o producción del objeto.

(iv) La estructura de tipificación léxica, que explica cómo se relaciona una palabra con otra en el lexicón mental.

Dentro de este marco, se presta especial atención a las entidades denominadas tipos complejos (dotted types), los cuales, se definen como palabras polisémicas que contienen dos sentidos en simultáneo en su rol formal y, por lo tanto, hacen posibles diferentes interpretaciones según el contexto sintáctico en el que aparecen. En este grupo se pueden observar dos clases, tipos complejos endocéntricos y tipos complejos exocéntricos (Pustejovsky, 1995; Adelstein \& Berri, 2008; Berri, 2014). Los tipos complejos endocéntricos permiten que se actualicen simultáneamente, como, por ejemplo, 'libro', que contiene la cualidad de objeto físico y la de información:

(10) Juan escribió un libro de historia_información que pesa dos kilos_objetofísico.

Los tipos complejos exocéntricos, por su parte, no pueden actualizar las distintas facetas a las que aluden. En Adelstein y Berri (2008) se ejemplifica con 'diario', que no puede referir al lugar en donde se producen los diarios y al objeto físico al mismo tiempo.

En relación con ello, en Pustejovsky (1995) se caracteriza la estructura de estos como productos cartesianos de los tipos naturales y los artificiales:

$t_{1} X t_{2}$

En donde el resultado es el par ordenado $<t_{1}, t_{2}>$, y donde $t_{1}=t_{1} \mathrm{y} t_{2}=t_{2}$ (Pustejovsky, 1995; Romero, 2015).

A su vez, existe una relación $R$ entre $t_{1}$ y $t_{2}, \mathrm{R}\left(t_{1}, t_{2}\right)$, dicha relación se establece en el propio ítem. En Rumshisky et al. (2007) se ofrece una lista de los principales nombres de tipo complejo, junto con algunos ejemplos:

- Acción•Propuesta ('promesa', 'alegación', 'mentira', 'cargo')

- Estado•Proposición ('creencia')

- Atributo•Valor ('temperatura', 'peso', 'altura', 'tensión', 'fuerza') 
- Evento•(Información•Sonido) ('concierto', 'sonata', 'sinfonía', 'canción')

- Información•Objeto Físico ('artículo', 'libro', 'CD', 'DVD', 'diccionario', 'diario', 'e-mail', 'ensayo', 'carta', 'novela', 'documento')

- Organización•(Información•Objeto Físico) ('periódico’, 'revista’, ‘boletín')

- Organización•Ubicación•Grupo Humano ('universidad', 'ciudad')

- Evento•Ubicación•Grupo Humano ('clase')

- Apertura•Objeto Físico ('puerta', 'ventana')

- Proceso•Resultado ('construcción', 'imitación', 'retrato', 'referencia', 'decoración', 'exhibición', 'documentación', 'dibujo', 'instrucción', 'invento', 'simulación', 'ilustración', 'acuerdo', 'aprobación', 'reconocimiento', 'daño', 'compensación', 'contribución', 'descuento', ‘donación', 'adquisición', 'clasificación', 'compra')

- Productor•Producto ('Honda', 'IBM', 'BMW')

- Árbol•Fruto /Arbol•Madera ('manzana', 'naranja', 'café' / 'roble', 'olmo', 'pino')

- Animal•Alimento ('anchoa', 'barbo', 'pollo', 'anguila', 'arenque', 'cordero', 'pulpo', 'conejo', 'calamar', 'trucha')

- Contenedor ${ }^{\bullet}$ Contenido ('botella', 'cubo', 'bote', 'caja', 'taza', 'frasco', 'barril', 'cazo', 'cuchara')

Al respecto, se pretende establecer una correlación entre la polisemia presentada por los NEND y las clases de objeto de la LG.

\section{Metodología}

Se analizó una lista de NEND extraída de la subsección Ginecología y Obstetricia del corpus CCM-2009, compilado por Burdiles (2012). La selección de las unidades de análisis a partir de corpus se justifica, en primer lugar, en que permite contar con un número adecuado de unidades a analizar y, en segundo lugar, en que un dominio de conocimiento se presenta como una parcela organizada del sistema de la lengua (Lamiroy, 1991), lo que hace más sistemático el uso de la terminología específica. El CCM-2009 está compuesto por casos clínicos publicados en revistas de medicina indizadas en la plataforma Scielo y fue lematizado de manera automática en el marco del Proyecto FONDECyT 1171033 mediante el uso de la herramienta de análisis lingüístico NooJ (Silberztein, 2016). Para el presente trabajo, se extrajeron aquellas palabras con la etiqueta 'nombre' y, mediante una revisión manual, se seleccionaron los NEND; obteniendo, de esta manera 86 unidades, de las cuales se seleccionaron 39 para analizar.

Las etapas de la metodología fueron las siguientes: (i) elaboración de tablas LG de preferencias de selección léxica para los NEND; (ii) elaboración de tablas LG de posibilidades transformacionales de cada EA resultante del análisis distribucional de 
(i); (iii) análisis de las EA que seleccionan los NEND en calidad de argumentos, y (iv) elaboración de estructuras semánticas (ES), a partir de la información proporcionada en (i), (ii) y (iii), según los criterios de la TLG. A continuación se desarrollan dichos pasos.

\subsection{Selección de unidades a analizar y análisis distribucional}

La sección de textos del área de ginecología y obstetricia del corpus CCM-2009 está compuesta por 89 casos clínicos y suma un total de 188.000 palabras. Se reconocieron 86 NEND y se los clasificó en las siguientes categorías:

- Estados del Paciente: 'puerperio', 'enfermedad'

- Estudios: 'biometría', 'cistometría'

- Intervenciones Médicas: 'aborto', 'histerectomía', 'cirugía'

- Hallazgos: 'cáncer', 'disnea', 'metaplasia'

- Procesos fisiológicos: 'aborto', 'sangrado’

- Síntoma: 'fiebre'.

Para esta clasificación inicial, se tomó como base la propuesta en la ontología SNOMED CT (2013) y se adaptó a partir de consultas con expertos del dominio. De este modo, fue posible un primer ordenamiento de los NEND, que permitió la posterior elaboración de las tablas LG.

Es pertinente aclarar al respecto que algunos NEND podían pertenecer a más de una categoría, por ejemplo 'aborto', que puede considerarse tanto un proceso fisiológico (11.a) como un síntoma (11.b).

(11) a. La paciente presenta una hemorragia nasal.

b. La hemorragia es síntoma de aneurisma.

Cuando se presentó este tipo de casos, se optó por verificar las posibilidades de significado y observar si había una variación en la estructura argumental. En los ejemplos de (11), dado que en ambos casos remite al mismo significado y no presenta variación, se la incluyó solamente en las tablas de procesos fisiológicos.

No obstante, por motivos tanto de extensión como por no observarse los casos de polisemia que se pretende analizar, en el presente trabajo, no se tuvieron en cuenta los NEND ubicados en el grupo de los hallazgos ${ }^{2}$, de los síntomas, y de los procesos fisiológicos (con excepción de los que pueden considerarse procesos médicos). Así, la lista total de NEND analizados fue de 39 unidades, las cuales se indican en la Tabla 3: 
Tabla 3. Lista de NEND analizados.

\begin{tabular}{|c|c|c|}
\hline Estados & Estudios & Procesos médicos \\
\hline $\begin{array}{c}\text { 'embarazo', } \\
\text { 'enfermedad','puerperio' }\end{array}$ & $\begin{array}{l}\text { 'antibiograma', 'audiometría', } \\
\text { 'biometría', 'cervicometría', } \\
\text { 'cintigrama', 'cistometría', } \\
\text { 'cistometrograma', 'cistouretrograma', } \\
\text { 'citometría', 'colonoscopía', } \\
\text { 'ecocardiograma', 'electrocardiograma', } \\
\text { 'electroencefalograma', } \\
\text { 'espermiograma', 'espirometría', } \\
\text { 'fetometría', 'flujometría', 'gasometría', } \\
\text { 'hemograma', 'impedanciometría', } \\
\text { 'mielograma', 'oximetría', 'partograma' }\end{array}$ & $\begin{array}{l}\text { 'aborto', 'anastomosis', } \\
\text { 'anexectomía', } \\
\text { 'apendicectomía', 'cesárea', } \\
\text { 'cirugía', 'colectomía', } \\
\text { 'colostomía', 'colpectomía', } \\
\text { 'diverticulometría', } \\
\text { 'esfinterectomía', } \\
\text { 'histeroctomía', 'ilesostomía' }\end{array}$ \\
\hline
\end{tabular}

El análisis distribucional volcado en las tablas LG consistió en la especificación de (i) los argumentos seleccionados por el nombre, es decir, las clases de objeto involucradas; (ii) los verbos soportes que permitían la proyección de las EA, (iii) las preposiciones asociadas, y (iv) los elementos que podían acompañar el predicado nominal (determinante, modificadores, etcétera).

Para determinar las clases de objeto, se recurrió a la información proporcionada en el corpus CCM2009 (Burdiles, 2012); en casos de corresponder, se extendió la búsqueda al Corpus del Español (CREA) y se tuvo como informantes a expertos del dominio, a la vez que se consultó un diccionario especializado (RANM, 2012). Por ejemplo, para saber los casos argumentos exigidos por la unidad 'colpectomía', primero se buscó su aparición en el corpus, sin que se pudiera evidenciar estos:

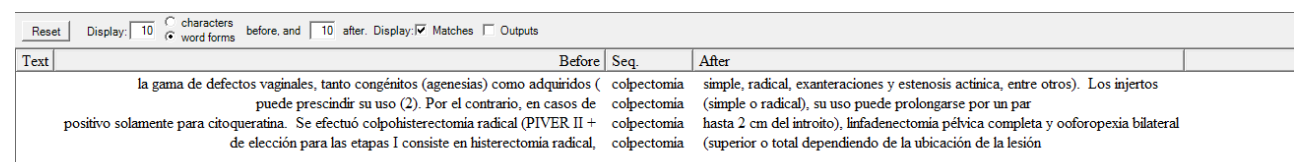

Figura 3. Resultados de búsqueda en el CCM-2009 de 'colpectomía'.

Posteriormente, se extendió la búsqueda al CREA sin obtener resultados. Esto implicó contar con el asesoramiento de expertos del domino, quienes informaron sobre el agente que lleva a cabo el evento, que en este caso es +Animado +Humano +Profesional de la Medicina y sobre qué o, más concretamente, sobre quién. Dado que se trata de una cirugía que consiste en la extirpación completa o de una parte de la vagina (RANM, 2012), el segundo argumento se debe corresponder con + Animado + Hembra +Paciente.

Para el análisis a nivel oracional, se recurrió a la introspección, la cual fue controlada por medio de los profesionales del dominio. 


\subsection{Análisis transformacional}

Se contemplaron las siguientes transformaciones:

- (T1) Reducción a SD: 01 La paciente tuvo un embarazo $\rightarrow 02$ El embarazo de la paciente.

- (T2) Voz pasiva: 01 El médico realizó una biopsia $\rightarrow 02$ Una biopsia fue realizada por el médico.

- (T3) Adjetivación del nombre: $01 \mathrm{El}$ puerperio de la paciente $\rightarrow 02$ La paciente está puerpérica.

- (T4) Verbalización del lema: 01 El radiólogo le hizo una radiografía a la paciente $\rightarrow 02 \mathrm{El}$ radiólogo radiografió a la paciente.

- (T5) Cambio de verbo soporte: $01 \mathrm{El}$ médico hizo una radiografía $\rightarrow 02 \mathrm{El}$ médico tuvo una radiografía.

- (T6) Cuantificación o pluralización del evento: 01 El médico hizo una cirugía $\rightarrow$ El médico hizo cinco cirugías.

Se ejemplifica con la oración 'El médico hizo una cesárea a la paciente'.

Tabla 4. Ejemplo de transformaciones para la oración 'El médico le hizo una cesárea a la paciente'.

\begin{tabular}{|l|l|l|l|l|l|l|}
\hline Oración & T1 & T2 & T3 & T4 & T5 & T6 \\
\hline El médico le hizo una cesárea a la paciente. & + & + & - & - & + & + \\
\hline
\end{tabular}

Mediante estas transformaciones se obtienen las siguientes oraciones:

(12) a. La cesárea de la paciente.

b. La cesárea fue hecha por el médico.

d. El médico hizo una cesárea.

e. El médico hizo cinco cesáreas (la semana pasada).

Si bien se podrían haber considerado otras transformaciones, las aquí presentadas permitieron la observación de la totalidad de los significados proyectados por los NEND. Asimismo, a los fines metodológicos, se optó por considerar las T5 y T6 en el análisis distribucional.

\subsection{Análisis de EA que seleccionan NEND como argumentos}

Finalmente, para otros significados posibles, se observaron en el corpus aquellas estructuras argumentales en las que los NEND participaban en calidad de argumentos. Esto permitió el reconocimiento de significados no proyectados por las unidades cuando son núcleo de la EA³. Para ello, además de la introspección y recurrencia a 
informantes expertos del dominio, se observó el comportamiento de las unidades léxicas a través del corpus.

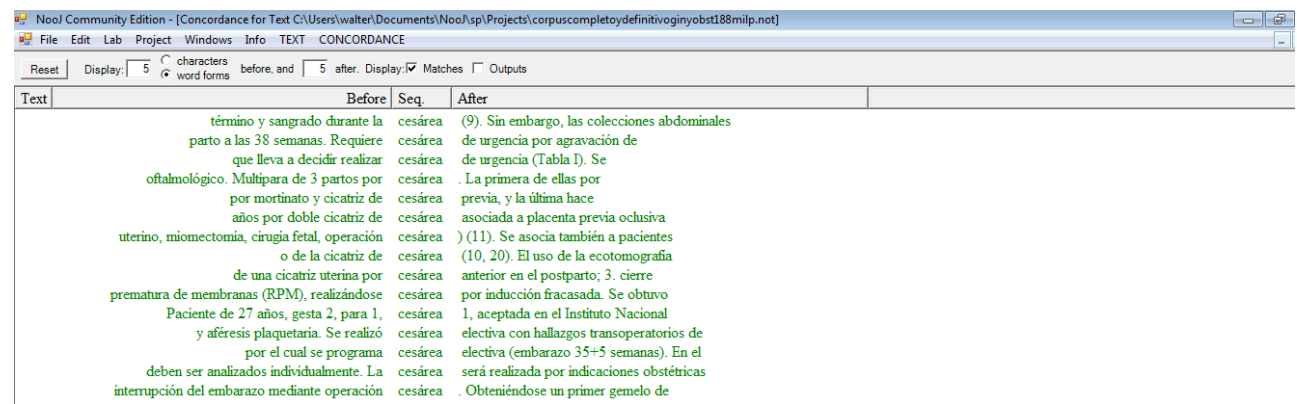

Figura 4. Fragmento del comportamiento en el corpus CCM2009 del término 'cesárea'.

El análisis de las EA que seleccionaban los NEND como argumentos permite dar cuenta, fundamentalmente, de lecturas no eventivas, como por ejemplo 'La paciente mostró la cesárea'.

\subsection{Elaboración de estructuras semánticas}

Finalmente, a fin de organizar la información reunida a través de las etapas precedentes, se propusieron estructuras semánticas (ES) de acuerdo con los postulados con la TLG. A continuación, se ejemplifica con 'embarazo'.

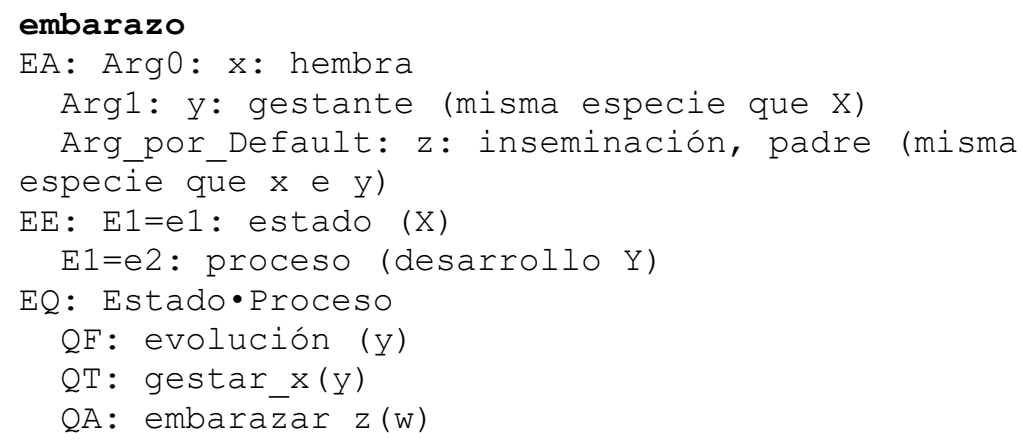

Figura 5. ES de 'embarazo'.

A continuación se presentan los resultados del análisis.

\section{Resultados}

\subsection{Estados del paciente: Estado•proceso}

Se encontraron 3 NEND pertenecientes a esta clase. Se pudo observar que estos seleccionan un primer argumento (Arg0) con la característica +Hum. A su vez, ‘embarazo' y ‘puerperio' restringen el paciente al género femenino (+Hembra) ${ }^{4}$. Esta 
clase de entradas presenta la variación estado •proceso. Por un lado, aluden a un estado que puede tener un paciente a partir de una condición específica de embarazo (13a), puerperio (14a) o enfermedad (15a) y, por otro lado, al proceso de desarrollo que tiene el gestante, en el caso de 'embarazo' (13.b); la regeneración del útero (14.b), en el caso de 'puerperio', y el desarrollo de la enfermedad (15.b).

(13) a. (...) La evolución del embarazo es fisiológico (...) (CCM-2009)

b. (...) Paciente de 23 años, portadora de su tercer embarazo, con antecedente de dos abortos previos. (...)(CCM-2009)

(14) a. La paciente cursa un puerperio fisiológico (...)(CCM-2009)

b. El puerperio finaliza con la regeneración del útero.

(15) a. (...) las pacientes portadoras de la enfermedad (...)(CCM-2009)

b. (...) La progresión natural de la enfermedad tiene un curso benigno (...) (CCM-2009)

A continuación se presenta la tabla LG para esta clase. 
Tabla 5. Tabla LG para 'estados del paciente'.

\begin{tabular}{|c|c|c|c|c|c|c|c|c|c|c|c|c|c|c|}
\hline \multirow{3}{*}{ Lema } & \multicolumn{3}{|c|}{ 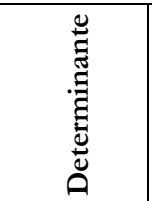 } & \multirow{3}{*}{ 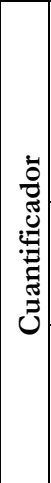 } & \multicolumn{2}{|c|}{ 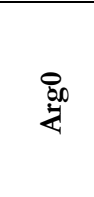 } & \multicolumn{3}{|c|}{ 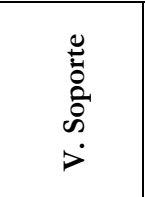 } & \multirow{3}{*}{ 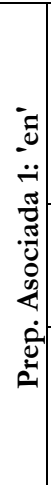 } & \multicolumn{2}{|c|}{ לab } & \multirow{3}{*}{ 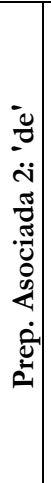 } & \multirow{3}{*}{ Ejemplo } \\
\hline & \multirow{2}{*}{ 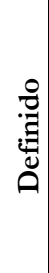 } & \multirow{2}{*}{ 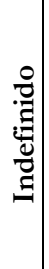 } & \multirow{2}{*}{ 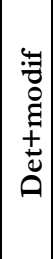 } & & \multicolumn{2}{|c|}{$\underset{+}{\stackrel{\Xi}{\Xi}}$} & \multirow{2}{*}{$\begin{array}{c}\dot{0} \\
\overrightarrow{0} \\
\stackrel{0}{-1}\end{array}$} & \multirow{2}{*}{ 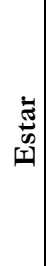 } & \multirow{2}{*}{ 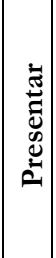 } & & \multicolumn{2}{|c|}{$\underset{+}{\stackrel{\Xi}{\Xi}}$} & & \\
\hline & & & & & 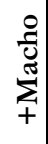 & 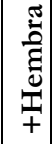 & & & & & $\begin{array}{l}\frac{0}{J} \\
\underset{+}{\mathbb{J}} \\
\end{array}$ & 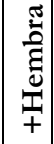 & & \\
\hline puerperio & + & - & - & - & - & + & - & + & - & + & - & - & - & La paciente está en puerperio. \\
\hline puerperio & + & - & - & - & - & + & + & - & - & - & - & - & - & La paciente tiene puerperio. \\
\hline puerperio & - & - & - & - & - & + & - & - & + & - & - & - & - & La paciente presenta puerperio. \\
\hline embarazo & + & + & - & - & - & + & + & - & - & - & + & + & + & $\begin{array}{l}\text { La paciente tiene un embarazo de } \\
\text { mellizos. }\end{array}$ \\
\hline embarazo & - & + & - & - & - & + & + & - & - & - & + & - & + & $\begin{array}{l}\text { La paciente tiene un embarazo de } \\
\text { su amante. }\end{array}$ \\
\hline embarazo & - & + & - & - & - & + & - & - & + & - & + & + & + & $\begin{array}{l}\text { La paciente presenta un embarazo } \\
\text { de mellizos. }\end{array}$ \\
\hline embarazo & - & + & - & - & - & + & - & - & + & - & + & - & + & $\begin{array}{l}\text { La paciente presenta un embarazo } \\
\text { de su amante. }\end{array}$ \\
\hline embarazo & + & - & - & - & - & + & - & + & - & + & - & + & - & La paciente está en embarazo. \\
\hline enfermedad & - & + & - & - & + & + & + & - & - & - & - & - & - & $\begin{array}{l}\text { El/la paciente tiene una } \\
\text { enfermedad. }\end{array}$ \\
\hline enfermedad & - & - & + & - & + & + & + & - & - & - & - & - & - & $\begin{array}{l}\text { El/la paciente tiene la enfermedad } \\
\text { de Huntington. }\end{array}$ \\
\hline enfermedad & - & - & - & + & + & + & - & - & - & - & - & - & - & $\begin{array}{l}\text { El/la paciente tiene dos } \\
\text { enfermedades. }\end{array}$ \\
\hline enfermedad & - & + & - & - & + & + & - & - & + & - & - & - & - & $\begin{array}{l}\text { El/la paciente presenta una } \\
\text { enfermedad. }\end{array}$ \\
\hline enfermedad & - & - & + & - & + & + & + & - & - & - & - & - & - & $\begin{array}{l}\text { El/la paciente presenta la } \\
\text { enfermedad de Huntington. }\end{array}$ \\
\hline enfermedad & - & - & - & + & + & + & - & - & - & - & - & - & - & $\begin{array}{l}\text { El/la paciente presenta dos } \\
\text { enfermedades. }\end{array}$ \\
\hline
\end{tabular}

En este caso, los verbos soportes involucrados en estos NEND son 'tener', 'estar' y 'presentar'. A su vez, el núcleo predicativo puede requerir una preposición asociada, como 'en' ('La paciente está en puerperio', 'La paciente está en embarazo'), también, en el caso de 'embarazo', para conectar con el segundo argumento, que puede ser el desarrollo del producto ('mellizos'), como, en este caso, el creador del embarazo (Quale agentivo).

La categoría Determinante más modificador (Deter+modif) es propuesta por Gross (2014) y refiere a los predicados se proyectan como complementos de un SD 
únicamente cuando tienen un modificador, sintagma adjetivo o sintagma preposicional.

(16) a. La paciente tiene la enfermedad de Huntington.

b.*La paciente tiene la enfermedad.

Esto se debe a que 'tener', en casos como estos, exige una predicación secundaria que afecta al segundo argumento. Así, en (16.a), se declara que la paciente tiene una enfermedad y que, además, esa enfermedad es la enfermedad Huntington.

Con respecto a las transformaciones, los tres términos permiten la transformación a SD (T1) (17); la adjetivación (T3) (18); y solo 'embarazo' y 'enfermedad' permiten la transformación a verbo (T4) (19).

(17) a El embarazo de la paciente.

b. El puerperio de la paciente.

c. La enfermedad de la paciente.

(18) a. La paciente está embarazada.

b. La paciente está puerpérica.

c. La paciente está enferma.

(19) a. La paciente se embarazó / Juan embarazó a María.

b. La paciente se enfermó.

En cuanto al comportamiento de estos NEND como argumentos, el Quale agentivo de 'embarazo' se manifiesta al ser esta unidad léxica seleccionada por la preposición 'por': 'el embarazo por inseminación (artificial)'.

En relación con el fenómeno de los tipos complejos, 'puerperio' y 'embarazo' presentan una complejidad en el Quale formal, como se puede ver en la Figura 6.

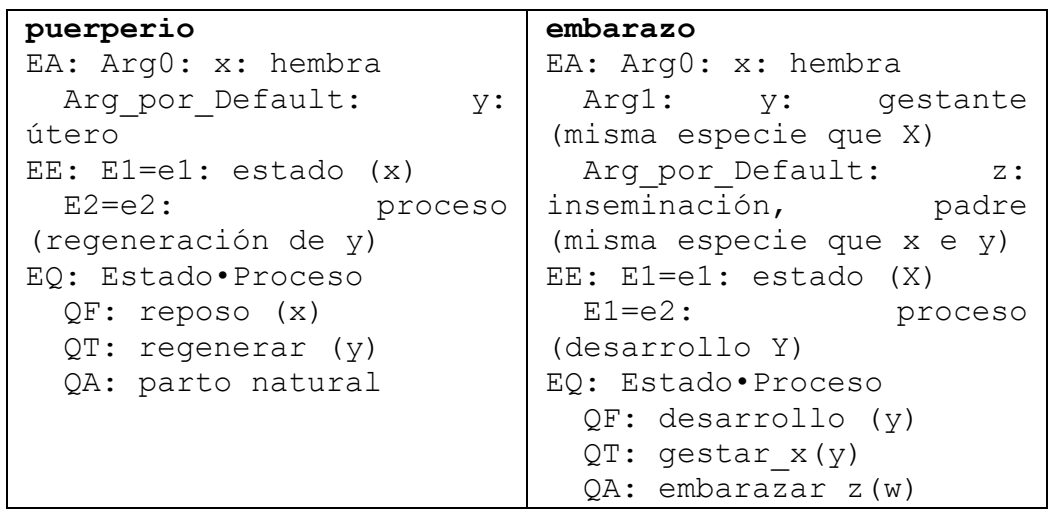

Figura 6. ES de 'puerperio’ y ‘embarazo’. 
Para dar cuenta de la complejidad de estos NEND, fue necesario incluir dentro de los tipos complejos la relación Estado•Proceso, no contemplada en la lista de Rumshisky et al. (2007). En este caso, se trataría de lo que en De Miguel y Fernández Lagunilla (2007) se denomina proceso de acabamiento gradual; es decir, un proceso que puede terminar en un logro (Zou, 2015) ${ }^{5}$.

\subsection{Estudios: Proceso•(Información•Objeto Físico)}

En el corpus se detectaron 23 NEND que se correspondían con estudios. La lectura eventiva se proyecta a partir de la selección de un primer argumento humano, que puede ser agente cuando se trata de un profesional de la medicina (+ProfMed) o paciente (+Pac). La polisemia que aquí se registra se corresponde con proceso•información•objeto físico. Esto se puede observar en que remite tanto al proceso de toma y análisis de muestras y el objeto físico que contiene la información. A modo de ejemplo, en el caso de 'biopsia' alude tanto a la extracción del fragmento a analizar, el análisis y el objeto físico que contiene la información.

(20) a. (...) Se toma biopsia que informa adenocarcinoma. (CCM-2009)

b. (...) Revisión de informes de biopsia archivados (...) (CCM-2009)

A continuación, y dado que las unidades presentaban un comportamiento similar, se ejemplifica con 'biopsia' y 'radiografía'. 
Tabla 6. Tabla LG para los NEND correspondiente con estudios (fragmento).

\begin{tabular}{|c|c|c|c|c|c|c|c|c|c|c|c|c|c|c|c|}
\hline \multirow[b]{2}{*}{ Estudios } & \multicolumn{3}{|l|}{ 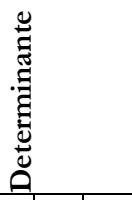 } & \multicolumn{2}{|c|}{ bo } & \multicolumn{3}{|l|}{ 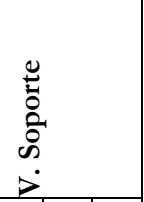 } & \multirow{2}{*}{ 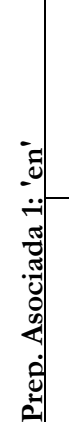 } & \multirow{2}{*}{ 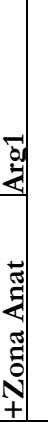 } & \multirow{2}{*}{\multicolumn{2}{|c|}{ 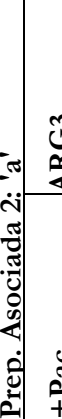 }} & |. & \multirow{2}{*}{ 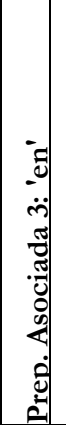 } & \multirow[b]{2}{*}{ Ejemplo } \\
\hline & 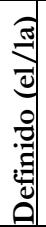 & 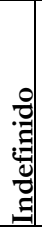 & 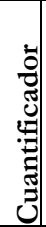 & $\begin{array}{c}0 \\
\overline{+} \\
\end{array}$ & $\begin{array}{l}0 \\
\sum_{0}^{0} \\
0 \\
0 \\
+\end{array}$ & 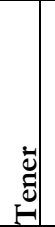 & 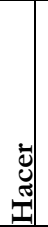 & 苟 & & & & & 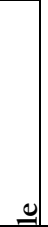 & & \\
\hline biopsia & + & + & + & + & - & + & - & - & - & - & -1 & - & - & - & $\begin{array}{l}\text { La paciente tiene una } \\
\text { biopsia. }\end{array}$ \\
\hline biopsia & + & + & + & - & + & - & + & - & - & - & - & - & $\#$ & - & $\begin{array}{l}\text { El médico le hizo una } \\
\text { biopsia a la paciente. }\end{array}$ \\
\hline biopsia & + & + & + & - & + & - & - & + & + & - & - & - & - & - & $\begin{array}{l}\text { El médico está en una } \\
\text { biopsia. }\end{array}$ \\
\hline
\end{tabular}

\begin{tabular}{|l|c|c|c|c|c|c|c|c|c|c|c|c|c|c|l|}
\hline radiografía & - & + & - & + & - & + & - & - & - & + & - & - & - & - & $\begin{array}{l}\text { La paciente tiene una } \\
\text { radiografía de tórax. }\end{array}$ \\
\hline radiografía & - & + & - & + & - & - & - & + & + & + & - & - & - & - & $\begin{array}{l}\text { La paciente está en una } \\
\text { radiografía de tórax. }\end{array}$ \\
\hline radiografía & - & + & - & - & + & - & + & - & - & + & + & + & $\#$ & - & $\begin{array}{l}\text { El médico le hizo una } \\
\text { radiografía de tórax a la } \\
\text { paciente. }\end{array}$ \\
\hline radiografía & - & + & - & - & + & - & - & + & + & + & - & - & - & + & $\begin{array}{l}\text { El médico está en una } \\
\text { radiografía de tórax. }\end{array}$ \\
\hline
\end{tabular}

Los verbos soportes que se tuvieron en cuenta fueron 'hacer', 'tener' y 'estar'. En cuanto a los argumentos seleccionados por el predicado, se consideró dos posibles, la zona anatómica en donde se realiza el estudio (+Zona Anat) y el paciente a quien se le realiza el estudio ( $+\mathrm{Pac})$. Con respecto al primero, este solo es seleccionado en aquellos casos en que el estudio tenía un carácter más general, es decir, se podía realizar en diferentes zonas corporales ('radiografía de tórax/de fémur'), mientras que aquellos que lo saturan desde la propia entrada léxica, por razones obvias, lo rechazan:

(21) *El enfermero le hizo un hemograma de sangre a la paciente.

$\mathrm{El}$ argumento que recibe caso dativo puede ser duplicado, por lo que se indica esta opción mediante el símbolo numeral (\#).

Ahora bien, estas entradas presentan las siguientes ES: 


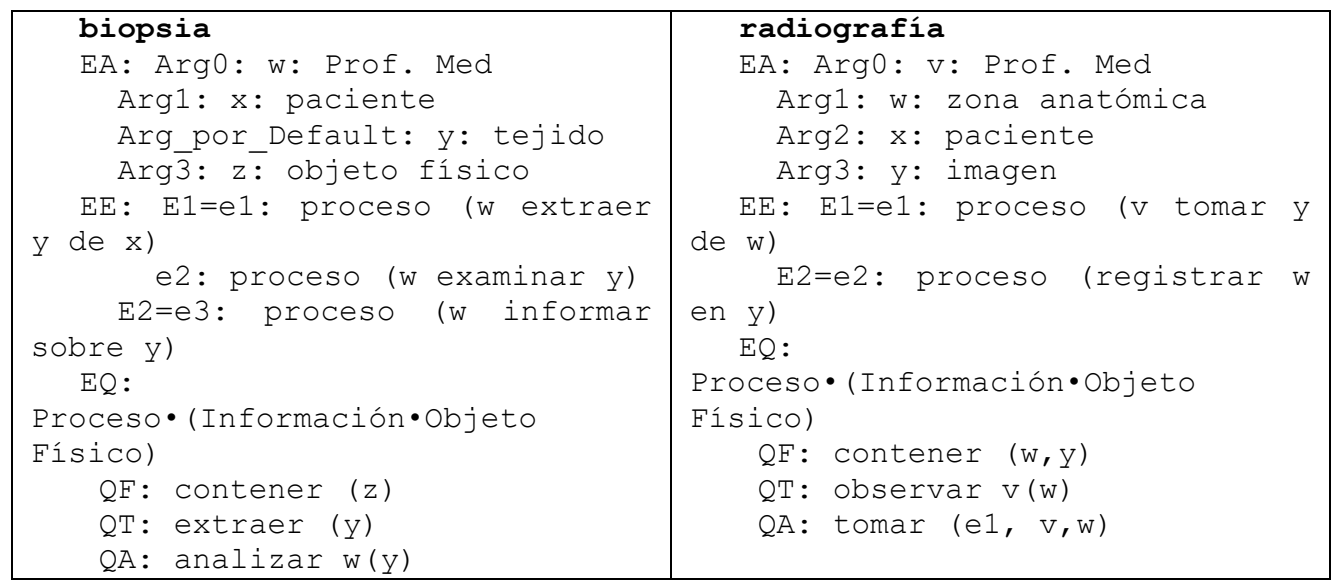

Figura 7. ES de 'biopsia' y 'radiografía'.

Se pudo apreciar que los estudios de carácter general, como 'radiografía', admiten una lectura no eventiva, de carácter locativo, al referir al lugar en donde estos se realizan.

(22) El médico está en radiografías.

En este caso, vale aclarar que es posible esta interpretación en ocasiones en que el nombre está seleccionado por la preposición 'en' y, en este caso, 'estar' deja de ser un verbo soporte, para pasar a predicar y seleccionar un argumento locativo mediante la preposición 'en', que se presenta como un sintagma nominal desnudo y al que no es posible incluirlo en la proyección de un SD:

(23) *El médico está en las radiografías.

Por otro lado, esta lectura no es permitida por otros tipos de estudio:

(24) *El médico está en biopsias.

Al respecto, podría decirse que en oraciones como las de (18) se activa el Quale agentivo en la medida en que alude al lugar donde se realizan estos estudios.

En este caso, y al igual que en para los estados del paciente, fue necesario la inclusión de una nueva relación: Proceso•(Información•Objeto Físico).

\subsection{Procesos médicos: Proceso •resultado}

Se detectaron 13 NEND de este tipo. Esta clase remite a las acciones llevadas a cabo por profesionales de la medicina (+ProfMed) sobre un paciente (+Pac). En algunos casos, estos procesos podían también coincidir con procesos fisiológicos, puesto que se pueden dar de manera natural (RANM, 2012), como 'anastomosis', los cuales rechazan 'ProfMed' como Arg0. La polisemia exhibida por esta clase de unidades remitía a proceso ${ }^{\circ}$ resultado. Se ejemplifica con 'tumorectomía' ( 7 de los 13 
se correspondían con NEND constituidos por el sufijo '-ectomía' -extirpación-), 'cesárea' y 'cirugía'.

Tabla 7. Ejemplo de tabla LG para Procesos Médicos (fragmento).

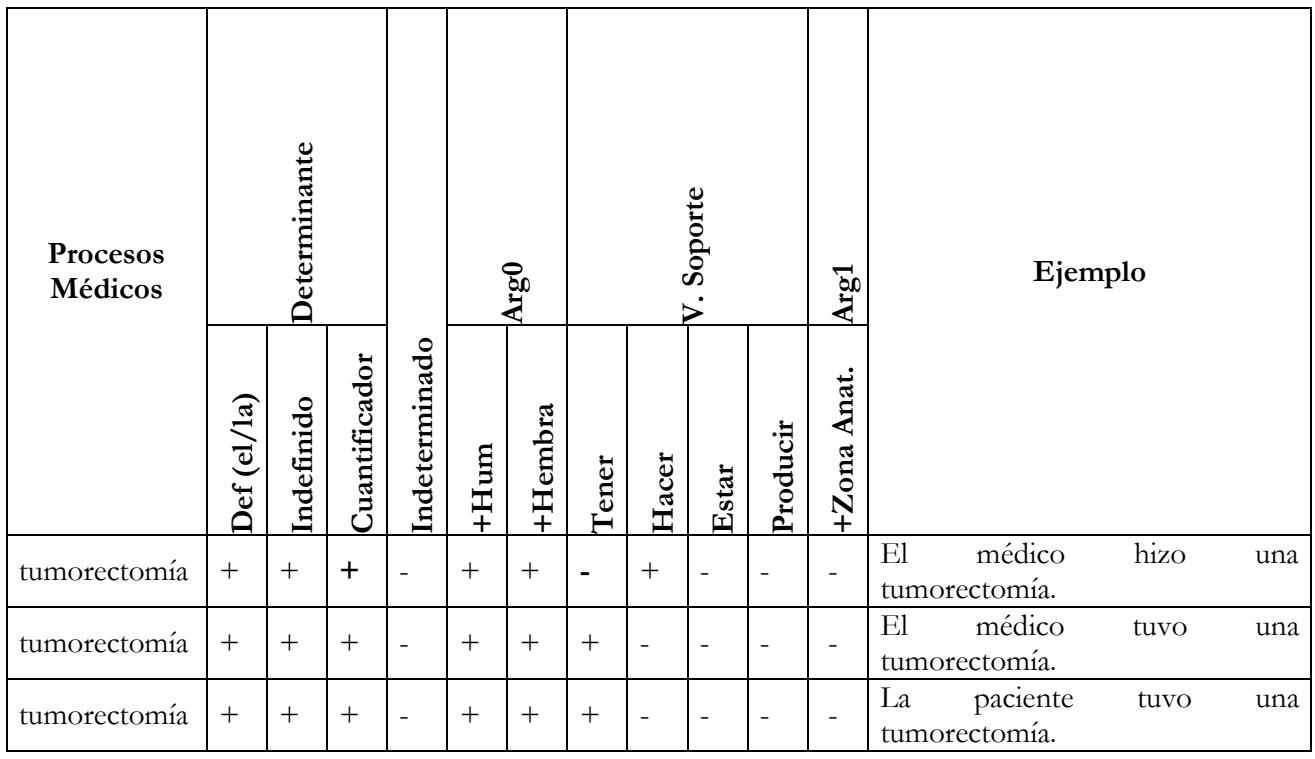

\begin{tabular}{|l|l|l|l|l|l|l|l|l|l|l|l|l|}
\hline cesárea & + & - & + & - & - & + & - & + & - & - & - & El médico hizo una cesárea. \\
\hline cesárea & + & - & + & - & - & + & + & - & - & - & - & El médico tuvo una cesárea. \\
\hline cesárea & + & + & - & - & - & + & - & - & + & - & - & El médico está en una cesárea. \\
\hline cesárea & + & - & + & - & - & + & + & - & - & - & - & La paciente tuvo una cesárea. \\
\hline cesárea & + & + & - & - & - & + & - & - & + & - & - & La paciente está en una cesárea. \\
\hline
\end{tabular}

\begin{tabular}{|l|l|l|l|l|l|l|l|l|l|l|l|l|}
\hline cirugía & + & - & + & - & + & - & - & + & - & - & + & El médico hizo una cirugía. \\
\hline cirugía & + & - & + & - & + & - & + & - & - & - & + & El médico tuvo una cirugía. \\
\hline cirugía & + & + & - & - & + & - & - & - & + & - & + & El médico está en una cirugía. \\
\hline cirugía & + & - & + & - & + & - & + & - & - & - & + & La paciente tuvo una cirugía. \\
\hline cirugía & + & + & - & - & + & - & - & - & + & - & + & La paciente está en una cirugía. \\
\hline
\end{tabular}

Las posibilidades transformacionales de cuantificación, permite ver la diferencia de significado en las oraciones de (25):

(25) a. 01: El médico tuvo una cesárea. $\rightarrow$ 02: El médico tuvo cinco cesáreas (en la semana).

b. 01: La paciente tuvo una cesárea. $\rightarrow$ 02: $*$ La paciente tuvo cinco cesáreas (en la semana). 
A la vez, otra de las transformaciones útiles para ver las diferencias de significado radica en las restricciones para el cambio del verbo soporte:

(26) a. 01: El médico tuvo una cesárea. $\rightarrow$ 02: El médico hizo una cesárea.

b. 01: La paciente tuvo una cesárea. $\rightarrow$ 02: *La paciente hizo una cesárea.

Por otro lado, el producto de la cesárea se explicita cuando la unidad léxica actúa como argumento de una preposición 'por' y el SP actúa en calidad de adjunto:

(27) La paciente tuvo un hijo por cesárea.

La lectura no eventiva se observó en casos en los que los NEND eran seleccionados por verbos del tipo 'mostrar' (28), lo que da cuenta del estado obtenido por la paciente.

(28) La paciente mostró la cesárea a su ginecólogo.

Una vez analizados los datos del análisis distribucional y de las apariciones de las unidades léxicas en el corpus, se proponen las siguientes ES:

\begin{tabular}{|c|c|c|}
\hline tumorectomía & cesárea & cirugía \\
\hline $\begin{array}{l}\text { EA: Arg0: x: Prof. } \\
\text { Med } \\
\text { Arg1: y: paciente } \\
\text { Arg_en_la_Som: } z: \\
\text { tumor } \\
\text { EE: E1=e1: proceso (x } \\
\text { extraer z de y) } \\
\text { E2=e2: estado (y } \\
\text { operado) } \\
\text { =e3: estado } \\
\text { extraído) } \\
\text { EQ: Proceso•Resultado } \\
\text { QF: extraer (z) } \\
\text { QT: sanar (y) } \\
\text { QA: operar (x,y) }\end{array}$ & $\begin{array}{l}\text { EA: Arg0: x: Prof. } \\
\text { Med } \\
\text { Arg1: y: paciente } \\
\text { (+hembra) } \\
\text { Arg_en_la_Som: } z: \\
\text { feto } \\
\text { EE: E1=e1: proceso } \\
\text { (w extraer z de y) } \\
\text { E2=e2: estado (y } \\
\text { operada) } \\
\text { =e3: estado } \\
\text { nacido) } \\
\text { EQ: } \\
\text { Proceso•Resultado } \\
\text { QF: extraer (x, z) } \\
\text { QT: } \\
\text { nacimiento (z) hacer } \\
\text { QA: extraer (x, z) }\end{array}$ & $\begin{array}{l}\text { EA: Arg0: } x: \text { Prof. } \\
\text { Med } \\
\text { Arg1: y: paciente } \\
\text { Arg_en_la_Som: } \mathrm{z} \text { : } \\
\text { zona anatómica } \\
\text { EE: E1=e1: proceso (w } \\
\text { reparar/mejorar z de } \\
\text { x) } \\
\text { E2=e2: estado (y, } \\
\text { mejorado) } \\
\text { EQ: Proceso•Resultado } \\
\text { QF: reparar (z) } \\
\text { QT: mejorar (y) } \\
\text { QA: sanar (x,y) }\end{array}$ \\
\hline
\end{tabular}

Figura 8. ES para 'tumorectomía', 'cesárea' y 'cirugía'.

De este modo, se observa que algunos significados de las unidades léxicas no se vislumbran en el análisis distribucional, cuando estas actúan en calidad de núcleo y es pertinente considerar más que la oración simple, como señala Gross (1975) en el inicio de su propuesta, la estructura argumental. En relación con esto último, en trabajos anteriores (Koza, 2017), se realiza un estudio de tablas LG para las preposiciones temporales, lo que sería de utilidad para las posibilidades y restricciones de significado de los NEND. 
A todo esto, es pertinente recordar lo mencionado más arriba respecto de las posibilidades de las entradas léxicas de pertenecer a más de una categoría. En relación con ello, debe aclararse que esta, en algunos casos, se justifica solamente a los fines clasificatorios del dominio médico, es decir, no se trataría, necesariamente, de polisemia. A modo de ejemplo, se observó que 'hemorragia' puede ser clasificada tanto como un proceso fisiológico como un síntoma; sin embargo, en ambos casos se alude al mismo evento, por lo cual no habría variaciones en el significado. No obstante, si pueden apreciarse diferentes comportamientos sintácticos en los tipos de proceso. Concretamente, el proceso médico implica la necesidad de un agente que desencadene el proceso, lo que no es necesario cuando esto ocurre, precisamente, de manera natural, como por ejemplo 'aborto' en 'aborto quirúrgico' y 'aborto espontáneo'.

\section{CONCLUSIONES}

Se presentó una propuesta para el análisis de la polisemia de los NEND a partir de dos marcos, la LG (Gross, 1975) y la TLG (Pustejovsky, 1995). Esto implicó la compilación de una lista de estas unidades léxicas y su posterior análisis. Para ello, se eligió el dominio de la medicina y se recurrió al corpus CCM-2009 (Burdiles, 2012), que fue procesado de manera semiautomática y de allí se extrajeron 39 NEND correspondientes con 'estados de un paciente', 'estudios' y 'procesos médicos'. Posteriormente, se elaboraron tablas LG que reflejaban las preferencias de proyección léxica de estas unidades y las posibilidades transformacionales para cada EA resultante. Asimismo, se observó el comportamiento que los NEND podían tener cuando eran seleccionadas en calidad de argumentos por otros núcleos predicativos. Toda esa información fue organizada en ES específicas.

$\mathrm{Al}$ respecto, se pudo apreciar que, por un lado, las tablas LG permiten observar las posibilidades de proyección de las EA, según sea la forma en que se actualiza un predicado nominal. Para ello, se recurre a un verbo soporte, que aporta los rasgos de persona y número, a la vez que le asigna caso nominativo al sujeto. A la vez, las posibilidades transformacionales dan cuenta de los distintos significados establecidos. En el análisis realizado, las transformaciones de cambio de verbo soporte y la cuantificación de los NEND permitieron observar las diferencias de significado.

En cuanto a los argumentos seleccionados por esta clase de predicados, se detectaron 3: Profesionales de la medicina ('médico', 'radiólogo'), Pacientes y Zonas anatómicas ('tórax').

Ahora bien, se pudo apreciar que el análisis distribucional y el de las transformaciones que este proyecta son insuficientes para dar cuenta de los posibles significados de los NEND. Esto significa que los significados de los NEND van más allá de las posibilidades de proyección sintáctica del predicado. Concretamente, las lecturas no eventivas que estos pueden llegar a tener no se pudieron reconocer en esta 
etapa, lo que implicó la necesidad de observar su comportamiento como argumentos de otra predicación. Al respecto también se concluye que no pueden considerarse las unidades léxicas como eventivas o no eventivas a priori, sino que estas posibilidades surgen de la estructura sintáctica en la que participan; esto se justifica en el mecanismo de co-composición que permite la generación de este tipo de lecturas.

No obstante, la elaboración de ES a partir de la propuesta de la TLG permitió organizar los datos arrojados por el análisis inicial. De este modo, las posibilidades de lecturas eventivas y no eventivas quedan reflejadas en la estructura de Qualia. A la vez, otras de las ventajas de recurrir a este modelo es la posibilidad de dar cuenta de los argumentos a la sombra (shadow arguments), que, precisamente, por su condición, no suelen estar visibles en el análisis distribucional.

El presente trabajo pretende hacer un aporte doble. Por un lado, contribuiría a los estudios en el ámbito de la LG, a partir de una propuesta para los NEND para el español. Al respecto, se plantea la posibilidad de incluir tablas de análisis distribucional no necesariamente a partir de verbos, sino la estructura argumental, proyectada tanto en un sintagma verbal, como nominal, adjetivo o preposicional. Por otro lado, se pretende aportar a la TLG, proponiendo dos tipos de nombres complejos, no contemplados en la propuesta de Rumshisky et al. (2007) ni en la de Romero (2015); me refiero a 'Estado•Proceso' y a 'Proceso•(Información•Objeto Físico)', dicotomías presentadas por algunos de los NEND incluidos en la categoría Estados del Paciente y Estudios, respectivamente.

En lo que concierne a tareas futuras, se llevará a cabo el análisis de los Procesos Fisiológicos, a la vez que se pretende seguir indagando cómo operan los mecanismos generativos y cómo se podría combinar con el análisis de tablas de la LG. Esto podría suponer, por un lado, ampliar el análisis distribucional a adjuntos específicos -lo que supone, en principio, una combinación de estructuras argumentales (las proyectadas en los adjuntos y una EA principal)- y, por otro, establecer posibles combinaciones entre mecanismos y transformaciones.

\section{REFERENCIAS BIBLIOGRÁFICAS}

Adelstein, A. \& Berri, M. (2008). Polisemia regular en nombres concretos relacionales del dominio de la tecnología. En Actas del XV Congreso Internacional de la Asociación de Lingüística y Filología de América Latina. [CD-Rom] Montevideo: Universidad de la República.

Adelstein, A., Berri, M. \& Boschiroli, V. (2012). Polisemia regular y representación lexicográfica: los nombres locativos en español. Terminalia, 5, 33-41. 
Adelstein, A. \& Boschiroli, V. (2015). Estatuto terminológico de nombres abstractos meronímicos de referencia general: Descripción y propuesta de representación en diccionarios para la producción académica. Debate terminológico, 14, 30-54.

Alexiadou, A. (2001). Functional Structure in Nominals: Nominalization and Ergativity. Ámsterdam: John Benjamins.

Berri, M. (2012). Léxico Generativo y aplicaciones lexicográficas: Los nombres concretos del dominio de la medicina en el DRAE. Revista Signos. Estudios de Lingüistica, 46(82), 190-212.

Berri, M. (2014). Polisemia regular en nombres locativos del español. Tesis doctoral, Universidad de Buenos Aires, Buenos Aires, Argentina.

Blanco, X. (2016). A hierarchy of semantic labels for Spanish dictionaries. Communications in computer and information science, 667, 66-73.

Bosque, I. (1999). Sustantivos eventivos. En I. Bosque \& V. Demonte (Dirs.), Gramática descriptiva de la lengua española (pp. 51-53). Madrid: Espasa.

Burdiles, G. (2012). Descripción de la organización retórica del género Caso Clínico de la medicina a partir del corpus CCM-2009. Tesis Doctoral, Pontificia Universidad Católica de Valparaíso, Valparaíso, Chile.

Chomsky, N. (1970). Remarks on nominalizations. En R. Jacobs \& P. Rosenbaum (Eds.), Readings in English transformational grammar (pp. 184-221). Waltham: Ginn.

De Miguel, E. (2009). La Teoría del Lexicón Generativo. En E. De Miguel (Ed.), Panorama de la lexicología (pp. 337-368). Madrid: Ariel.

De Miguel, E. (2011). En qué consiste ser verbo de apoyo. En V. Escandell, M. Leonetti \& C. Sánchez (Eds.), 60 problemas de gramática (pp. 139-146). Madrid: Akal.

De Miguel, E. \& Fernández Lagunilla, M. (2007). Sobre la naturaleza léxica del aspecto composicional. Actas del VI Congreso de Lingüistica General (pp. 1767-1778). Madrid: Arco/Libros.

Elia, A., Monteleone, M. \& Marano, F. (2011). From the concept of transformation in Harris and Chomsky to the Lexique-Grammaire of Maurice Gross. En V. Kasevich, Y. Kleiner \& P. Sériot (Eds.), History of linguistics (pp. 76-82). Ámsterdam: John Benjamins.

Fábregas, A. (2010). Los nombres de evento: Clasificación y propiedades en español. Pragmalingüistica, 18, 54-73. 
Grimshaw, J. (1990). Argument structure. Cambridge, Massachusetts: MIT Press.

Gross, G. (2014). Manual de análisis lingüistico. Aproximación sintáctico-semántica al léxico. Barcelona: Editorial UOC.

Gross, M. (1975). Méthodes en syntaxe. París: Hermann.

Gross, M. (1998). Lexique, grammaires et cumulativité. Cahiers de l'institut de linguistique de Louvain, 24, 23-41.

Halle, M. \& Marantz, A. (1993). Distributed morphology and the pieces of inflection. En K. Hale \& J. Keyser (Eds.), The View from Building 20. Essays in Linguistics in Honor of Sylvain Bromberger (pp. 111-176). Cambridge, Massachussets: MIT Press.

Harris, Z. (1970). Papers in structural and transformational linguistics. Dordrecht: Springer.

Kocijan, K. \& Librenjak, S. (2016). Recognizing verb-based Croatian idiomatic MWUs. Communications in computer and information science, 667, 96-106.

Koza, W. (2017). Preposiciones temporales en español. Descripción y análisis sintáctico-semántico. Ponencia presentada en el I Coloquio de Gramática - Tiempo Aspecto y Modalidad: Expresión gramática y bases cognitivas, Universidad de Chile, Santiago, Chile.

Koza, W., Filippo, D., Cotik, V., Stricker, V., Muñoz, M., Rivas, N. Godoy, N. \& Martínez, R. (2018). Automatic detection of negated findings in radiological reports for Spanish language: Methodology based on Lexicon-Grammatical information processing. Journal of Digital Imaging. First Online.

Lamiroy, B. (1991). Léxico y gramática del español. Estructuras verbales de espacio y tiempo. Barcelona: Editorial Anthropos.

Langella, A. (2016). Paraphrases for the Italian comunicative predications. Communications in computer and information science, 811, 182-195.

Le Pesant, D. \& Mathieu-Colas, M. (1998). Introduction aux clases d'objets. Langages, 131, 6-33.

Messina, S. \& Langella, A. (2015). Paraphrases $\mathrm{V} \leftrightarrow \mathrm{N} \leftrightarrow \mathrm{A}$ in one class of Psychological Predicates. En J. Monti, M. Silberztein, M. Monteleone \& M. di Buono (Eds.), Formalising Natural Languages with NooJ 2014 (pp. 140-149). Newcastle: Cambridge.

Mota, C., Barreiro, A., Raposo, F., Ribeiro, R., Curto, S. \& Coheur, L. (2016). eSPERTo's paraphrastic knowledge applied to question-answering and summarization. Communications in computer and information science, 811, 208-222. 
Nazar, R., Soto, R. \& Urrejola, K. (2017). Detección automática de nombres eventivos no deverbales: un enfoque cuantitativo basado en corpus. Linguamática, 9(2), 21-33.

Palma, S. (1994). Hacia un enfoque semántico de las expresiones idiomáticas. En J. Coursera, M. Djian \& A. Gaspar (Eds.), La lingüistica francesa. Situación y perspectivas a finales del siglo XX (pp. 313-321). Zaragoza: Prensas de la Universidad de Zaragoza.

Papadopoulou, E. (2010). Diccionario monolingüe coordinado para enseñanal aprendizaje del griego moderno por parte de hispanohablantes y para traducción automática griego español. Tesis doctoral, Universidad Autónoma de Barcelona, Barcelona, España.

Pivaut, L. (1989). Verbes supports et vocabulaire technique: Sport, musique et activités intelectuells. Tesis doctoral, Universidad de París, París, Francia.

Pustejovsky, J. (1995) The generative lexicon. Cambridge, Massachusetts: MIT Press.

Pustejovsky, J. (2011). Coercion in a general theory of argument selection. Linguistics, $49(6), 1401-1431$.

Pustejovsky, J. (2013). Type theory and lexical decomposition. En J. Pustejovsky, J. Bouillon, P. Isahara, H. Hanzaki \& C. Lee (Eds.), Advances in Generative Lexicon Theory (pp. 9-38). Nueva York: Springer.

RANM (2012). Diccionario de términos médicos. Buenos Aires: Editorial Médica Panamericana.

Resnik, G. (2010). Los nombres eventivos no deverbales en español. Tesis doctoral, Universidad Pompeu Fabra, Barcelona, España.

Romero, J. (2015). Propuesta de clasificación de algunos nombres complejos en el Lexicón Generativo. Journal of foreign studies, 65(3), 53-69.

Rumshisky, A., Grinberg, V. \& Pustejovsky, J. (2007). Detecting selectional behavior of complex types in texts. Ponencia presentada en el 4th International Workshop on Generative Approaches to the Lexicon. París: Francia.

Silberztein, M. (2016). Formalizing natural languages. The NooJ approach. Londres: ISTE.

SNOMED CT (2013). Guía del usuario [en línea] Disponible en: http://www.snomed.org/doc

Subirats, C. (1987). Sentential complementation in Spanish. Amsterdam: John Benjamins.

Tolone, E. (2009). Les tabes du Lexique-Grammaire au format TAL. Ponencia presentada en Majecs'TIC 2009, Avignon, Francia. 
Tolone, E. (2012). Conversión de las tablas del Léxico-Gramática del francés en el léxico LGLex. Ponencia presentada en el 2nd Argentinian Workshop on Natural Language Processing (WNLP’11), Córdoba, Argentina.

Zou, X. (2015). El aspecto en chino. Clases de eventos y operadores aspectuales. Tesis doctoral, Universidad Autónoma de Madrid, Madrid, España.

\section{NOTAS}

${ }^{1}$ La LG adopta la noción de transformación propuesta por Harris (1970), y se corresponde con un dispositivo experimental que manifiesta las características sintácticas pertinentes de una estructura determinada mediante frases semántica y morfológicamente análogas (Lamiroy, 1991). Se diferencia de la noción chomskiana tradicional en la medida en que las transformaciones se dan todas en el plano de la estructura superficial.

2 Excepto 'enfermedad', que puede ser considerado un genérico, que se incluyó dentro de los estados.

${ }^{3}$ Esto implicaría, a su vez, la confección de tablas LG para dichos núcleos predicativos.

${ }^{4}$ En casos como 'enfermedad', que puede afectar tanto a un macho como a un hembra, se seleccionaron positivamente $(+)$ ambas opciones.

${ }^{5}$ Para una revisión a la noción de eventos en la TLG, Zou (2015) y De Miguel y Fernández Lagunilla (2007).

\section{AGRADECIMIENTOS}

* Esta investigación fue financiada por una subvención del Proyecto FONDECyT 1171033, de la Comisión Nacional Científico y Tecnológica (CONICy'T), Chile. 